\title{
Emissões otoacústicas como instrumento de vigilância epidemiológica na saúde do trabalhador
}

\section{Otoacoustic emissions as an instrument of epidemiological surveillance in the health of the workers}

\author{
Priscila Feliciano de Oliveira', Oscar Felipe Falcão Raposo², Any Caroline Aragão dos Santos ${ }^{3}$, Luana Araujo dos Santos ${ }^{4}$. \\ 1) Mestre em Fonoaudiologia pela PUC SP. Professora Assistente do Núcleo de Fonoaudiologia da Universidade Federal de Sergipe. \\ 2) Mestrado em Biometria e Estatística Aplicada pela UFRPE. Professor Assistente do Departamento de Estatística e Ciências Atuariais da Universidade Federal de Sergipe. \\ 3) Graduação em Fonoaudiologia pela Universidade Federal de Fonoaudiologia. Fonoaudióloga autônoma. \\ 4) Graduação em Fonoaudiologia pela Universidade Federal de Sergipe. Fonoaudióloga autônoma. \\ Instituição: Universidade Federal de Sergipe \\ São Cristóvão / SE - Brasil. \\ Endereço para correspondência: Priscila Feliciano de Oliveira - Universidade Federal de Sergipe - Centro de Ciências Biológicas e da Saúde - Av. Marechal Rondon, $\mathrm{s}$ n - \\ Jd Rosa Elze - São Cristóvão / SE - Brasil - CEP: 49100-000 - URL da Homepage: wwww.ufs.br - Telefone: (+55 79) 21056660 - E-mail: oliveirapriscila@ hotmail.com \\ Artigo recebido em 19 de Março de 2011. Artigo aprovado em 14 de Agosto de 2011
}

\section{RESUMO}

Introdução: O ruído é um agente nocivo à audição sendo frequente nos ambientes urbanos e laborativos. Dentre as estruturas do sistema auditivo, as células ciliadas externas são as primeiras a serem lesadas e as emissões otoacústicas identificam mínimas alterações cocleares.

Objetivo: Analisar alterações cocleares com as otoemissões acústicas evocadas transientes em indivíduos expostos ao risco combinado: ruído e produtos químicos.

Método: Participaram da pesquisa 49 trabalhadores de uma empresa de cimento, com idade entre 19 e 49 anos, tempo de exposição de no mínimo dois anos e limiares auditivos normais. Foi realizadas anamnese e emissões otoacústicas antes e pós-atividade laborativa. Os resultados do exame foram relacionados com as variáveis: tempo de exposição ao ruído, idade, exposição a produtos químicos e hábitos sonoros. Os testes estatísticos utilizados foram: T de Student, qui-quadrado de Pearson e Exato Fisher e caracteriza-se por um estudo clínico prospectivo.

Resultados: $\mathrm{Na}$ primeira testagem houve presença de emissões em todos os trabalhadores, a média de amplitude de 10,22 dBSPL na orelha direita e 9,48 dBSPL na orelha esquerda. Na segunda testagem houve uma variação de 0,69 dBSPL na orelha esquerda e $0,42 \mathrm{dBSPL}$ na orelha direita, sendo que $79,6 \%$ dos indivíduos tiveram presença de emissões bilateralmente e $20,4 \%$ ausência em pelo menos uma orelha. Ao analisar a relação entre variação de emissões com as variáveis, não se observou dado estatisticamente significante.

Conclusão: As emissões otoacústicas na saúde do trabalhador buscam prevenir o dano ao sistema auditivo através das alterações cocleares.

Palavras-chave: ruído ocupacional, serviços de saúde do trabalhador, testes auditivos.

\section{SUMMARY}

Introduction: The noise is a harmful agent to the hearing, being frequent in urban and work environments. Among the structures of the hearing system, the outer hair cells are the first to be injured, and otoacoustic emissions identify minimal cochlear alterations.

Objective: Analyze cochlear alterations with otoacoustic emissions transient evoked in individuals exposed to combined risk: noise and chemical products.

Method: 49 workers of a cement company participated of the research, aged between 19 and 49 years old, exposure time of at least two years and normal hearing thresholds. Was performed an anamnesis and otoacoustic emissions before and post work activity. The results of the exam were related with the variable: time of exposure to the noise, age, exposure to chemical products and sound habits. The statistical tests used were: T of Student, chi-squared Pearson test and Fisher's exact test and is characterized by a prospective clinical study. Results: At the first testing, had presence of emissions in all of the workers. The average of amplitude is of 10,22 dBSPL in the right ear and 9,48 dBSPL in the left ear. In the second testing there were a variation of $0,69 \mathrm{dBSPL}$ in the lef ear and $0,42 \mathrm{dBSPL}$ in the right ear, of which $79,6 \%$ of individuals had presence of emission bilaterally and $20,4 \%$ absence in at least one ear. Analyzing the relation between variations of emissions with the variable was not observed statistically significant data. Conclusion: The otoacoustic emissions in the workers health search to prevent the damage to the hearing system through cochlear changings.

Keywords: occupational noise, health worker services, hearing tests. 


\section{INTRODUÇÃO}

Há muito tempo, o ruído é considerado um agente nocivo à saúde do homem, sendo este cada vez mais frequente tanto nos ambientes urbanos quanto nas atividades laborativas. Sabe-se que os danos ocasionados à audição pelo ruído ocupacional são irreversíveis, a exemplo da perda auditiva induzida por ruído (PAIR).

A PAIR é uma diminuição gradual da audição devido à exposição a elevado nível de pressão sonora. Trata-se de uma patologia irreversível, neurossensorial e predominantemente coclear. Desenvolve-se gradualmente de forma mais rápida num período de 6 a 10 anos. Após esse tempo sua progressão torna-se mais lenta, tendendo a estabilizarse. As frequências mais atingidas são as agudas, sendo que caso a exposição seja eliminada, a evolução da perda auditiva também cessa (1).

Além de implicações auditivas, a PAIR também causa alterações extra auditivas, bem como alterações psicossociais caracterizadas por isolamento, estresse, dificuldade de sono, diminuição da auto- estima, depressão; além de transtornos neurológicos, vestibulares, digestivos, cardiovasculares e hormonais (2). Portanto, uma intervenção precoce nos trabalhadores expostos ao ruído.

Um dos meios de atuação preventiva é o monitoramento audiométrico que pode ser realizado pela avaliação auditiva (audiometria tonal, vocal eimitanciometria). Além destes exames básicos, com o advento das emissões otoacústicas (EOA) é possível a identificação de lesões antes mesmo de a perda auditiva ser instalada.

A aplicação das EOA, que é um exame objetivo, serve também de auxílio no diagnóstico diferencial da perda auditiva neurossensorial, e possibilita a identificação de mínimas alterações funcionais do sistema auditivo. As EOA são respostas de frequências específicas nas quais as células ciliadas externas (CCE) encontram-se normais ou próximas do normal, o que permite investigar simultaneamente diferentes partes da cóclea (3). Desse modo, acredita-se que as otoemissões presentes podem estar relacionadas à integridade das CCE (4).

Em pacientes expostos a elevado nível de pressão sonora, as EOA podem estar afetadas antes mesmo do aparecimento dos sintomas subjetivos e dos sinais audiométricos, demonstrando a agressão das CCEs, fato que revela as EOA como um exame complementar importante $(5,6)$.

Pensando nesses aspectos foi realizado um estudo com30 indivíduos com limiares auditivos normais do ramo de tecelagem. Todos os trabalhadores faziam o uso de proteção auricular e estavam expostos a ruído que alternava entre 80 a 90 dB NPS. A metodologia englobou audiometria tonal liminar e emissões otoacústicas evocadas transientes (EOAT) em cabina acústica, pré e pós-exposição a elevado nível de pressão sonora por 5 horas de trabalho. Na análise dos resultados das EOAT. Houve diminuição significativa da reprodutibilidade em todas as frequências pesquisadas na orelha direita e esquerda pósexposição ao ruído (5).

Com o intuito de verificar correlação entre mudança temporária de limiar auditivo, variações nas amplitudes de respostas das EOAE e susceptibilidade à perda auditiva induzida por ruído foram utilizado, na pesquisa, exames de EOA e audiometria. Fizeram parte do estudo dois grupos de trabalhadores: um com limiares auditivos normais e outro com diagnóstico de PAIR. Todos os exames foram realizados antes e após exposição a um ruído de 105 dB NA, por dez minutos, em cada uma das orelhas; por meio de fones em cabina acústica. Nas EOAET, as variações das amplitudes de resposta geral foi menor que $1 \mathrm{~dB}$, e as variações por banda de frequência, menor que $3 \mathrm{~dB}$. Não houve diferença significativa entre as orelhas direita e esquerda (7).

Um estudo comparativo foi realizado para confirmar a eficácia das EOAT com o intuito de correlacionar a audiometria tonal liminar, na avaliação de alterações cocleares decorrentes do ruído. Foram selecionados 32 trabalhadores do sexo masculino de uma fábrica de metal. Realizaram audiometria e EOAT pré e pós-jornada de trabalho, porém não foi verificada alteração significativa na audiometria tonal. Já as EOAT evidenciaram uma redução significativa após exposição ao ruído, com o valor médio de $1,2 \pm 1,1 \mathrm{~dB}$. Concluiu-se que as EOA são sensíveis na avaliação na coclear após a exposição ao ruído (8).

Uma população que está exposta ao elevado nível de pressão sonora são os trabalhadores da produção de cimento, objeto do nosso estudo. Além do ruído, também estão expostos a outro agente: a vibração, que pode interferir de forma negativa na saúde auditiva. A exposição concomitante: ruído e vibração podem agravar ainda mais os efeitos causados na audição, bem como alterar o bemestar físico e mental. Desta forma, o objetivo do presente estudo é analisar as respostas das otoemissões acústicas evocadas transientes em indivíduos expostos a ruído ocupacional.

\section{MÉTODO}

Este estudo teve delineamento epidemiológico do tipo transversal e foi aprovado pelo Comitê de Ética e aprovado com o número CAAE-3601.0.000.107-10 (Anexo). 
A amostra foi composta por 49 sujeitos com limiares auditivos normais de uma empresa do ramo de produção de cimentos. Foram incluídos na pesquisa trabalhadores do gênero masculino e feminino, porém a maioria da população pesquisada é do sexo masculino, uma vez que é quase a totalidade da população que atua no segmento. Os trabalhadores que participaram da pesquisa estavam expostos a um nível de ruído acima de $85 \mathrm{~dB}$ NPS e faziam o uso de proteção auricular. Também tinham histórico de exposição a ruído ocupacional a pelo menos dois anos por uma jornada de 8 horas diárias.

As audiometrias foram fornecidas pelo serviço de saúde da empresa e foram selecionados apenas os audiogramas que apresentaram limiares compatíveis com os padrões de normalidade nas duas orelhas. Os exames foram realizados em umúnico serviço por um fonoaudiólogo, sempre com o mesmo audiometro.

Após a seleção dos sujeitos de forma aleatória, eles foram convidados a preencherem uma anamnese que foi elaborada pelas pesquisadoras. Os dados da anamnese tinham a finalidade de obter informações sobre o tempo de exposição ao ruído, uso de proteção auricular, presença de zumbido e informações sobre a saúde em geral.

Em seguida foi realizado o exame de EOAT antes da jornada de trabalho com um repouso acústico de 14 horas. A testagem foi realizada na própria empresa, dentro de uma cabine acústica, que obedecia às normas da ANSI. Tal fato garantiu que o ruído de fundo não interferisse na testagem. Foi utilizado o equipamento manual de EOAE OTOPORT LITE, número de série OPL 09101991, fabricado pela Otodynamics LTD. Como parâmetro, o equipamento de EOAT em questão produz clique não linear como estímulo, apresentado em uma intensidade de $84 \mathrm{~dB}$ PK, para avaliar as bandas de meia-oitava centralizadas nas frequências 1,0; 1,$5 ; 2,0 ; 3,0$ e $4 \mathrm{kHz}$. Foram utilizados 260 cliques com um tempo máximo de testagem de 300 segundos. Os critérios utilizados se baseiam nos parâmetros de configuração do equipamento que consta no manual do fabricante.

Em uma terceira etapa da pesquisa foi realizada a repetição da EOAT pós-jornada de trabalho de 08 horas diárias. A testagem foi realizada em um único dia, sendo as EOAT pós-jornada realizada no máximo 15 minutos após o encerramento da atividade laborativa. O trabalhador foi convidado a retornar a sala que realizou a primeira testagem, após a jornada de trabalho para realização da segunda testagem com as EOAT.

A classificação e interpretação dos resultados foram feitas de acordo com piora, melhora ou manutenção da amplitude de resposta das EOAT, comparando-se os exames realizados antes e após a exposição ao ruído.
Após a finalização da avaliação foi criado um banco de dados com o software Statistical Package for the Social Sciences(SPSS, versão 17.0) para posterior análise estatística. Para a caracterização da população foi utilizada estatística descritiva (média, mediana, desvio padrão, máximo e mínimo) e para avaliar a relação entre as variáveis independentes (idade, exposição a produtos químicos, tempo de exposição ao ruído, hábitos sonoros e zumbido) foi realizado os testes $\mathrm{T}$ de Student, qui-quadrado de Pearson e ExatoFisher. Foram considerados estatisticamente significantes valores de p menores ou iguais a 0,05.

\section{RESULTADOS}

Os trabalhadores apresentaram idade média de 31,2 anos e o tempo de exposição ao ruído ocupacional variou de 2 a 28 anos, sendo que 42,85\% dos trabalhadores ficaram expostos a elevado nível de pressão sonora por um período de tempo de 02 a 05 anos.

Com relação ao produto químico, apenas 13 relataram exposição a algum agente 30 trabalhadores informaram não realizar nenhuma atividade que envolvesse exposição a elevado nível de pressão sonora. Quanto ao zumbido, 44 (89,8\%) trabalhadores não apresentaram queixa e apenas cinco $(10,2 \%)$ a relataram, sendo este do tipo apito ou chiado.

Com relação à avaliação das EOAT, todos os trabalhadores apresentaram EOAT presentes na primeira testagem, sendo esta caracterizada, conforme descrito na Tabela 1.

$\mathrm{Na}$ segunda testagem, entre os 49 trabalhadores envolvidos na pesquisa, 09 deles apresentaram ausência de EOAT na orelha direita e cinco na orelha esquerda, como pode ser observado na Tabela 2 .

Tabela I. Distribuição das características da amplitude das EOA nas orelhas direita e esquerda, na primeira testagem.

\begin{tabular}{lcc}
\hline Amplitude geral dasEOAT & $\mathrm{OD}(\mathrm{dB})$ & $\mathrm{OE}(\mathrm{dB})$ \\
\hline Média & 10,22 & 9,48 \\
Máximo & 23,00 & 19,80 \\
Mínimo & 4,20 & 1,90 \\
Desvio padrão & 3,61 & 3,98 \\
\hline
\end{tabular}

Tabela 2. Distribuição dos trabalhadores, segundo a presença e ausência de EOAT nas orelhas direita e esquerda $(n=49)$.

\begin{tabular}{lcc}
\hline EOAT & OD & OE \\
\hline Presença & $40(81,64 \%)$ & $44(89,8 \%)$ \\
Ausência & $9(18,36 \%)$ & $5(10,2 \%)$ \\
\hline Total & $49(100 \%)$ & $49(100 \%)$ \\
\hline
\end{tabular}


$\mathrm{Na}$ análise da segunda testagem, 39 (70,6\%) apresentaram EOAT presentes em ambas as orelhas, $04(8,2 \%)$ apresentaram ausência bilateralmente e $06(12,2 \%)$ em apenas uma das orelhas, desse modo 10 dos sujeitos envolvidos nessa pesquisa tiveram ausência de EOAT em pelo menos uma das orelhas

Considerando a amplitude média da orelha direita de 10,22 dB na primeira testagem e 9,80 dB na segunda testagem, é possível observar maior variação da amplitude geral na OE como pode ser observado na Tabela 3.

Correlacionando a variável dependente (EOAT) com as independentes não se observou valores estatisticamente significantes.

\section{DISCUSSÃO}

Conforme descrito nos resultados, a população pesquisada foi submetida à realização das EOAT em duas etapas, sendo que na primeira testagem 100\% dos trabalhadores apresentaram EOAT presentes. Em contraposição, a literatura relata que existem casos de ausência de reposta para EOAT mesmo nos indivíduos com limares auditivos normais $(5,6,7,8,9,10,11)$.

Na primeira testagem, antes da jornada de trabalho, o resultado da amplitude geral das EOAT, na orelha direita, foi de $23 \mathrm{~dB}[\mathrm{AC} 1]$ de amplitude máxima e 4,2 dB de mínima. Já na orelha esquerda, a amplitude máxima foi de $19,80 \mathrm{~dB}$ e mínima de $1,90 \mathrm{~dB}$ o que evidenciou maior amplitude máxima na orelha direita. Esses dados corroboram com estudos que também relataram maior amplitude na orelha direita $(6,10)$. Tal fato pode ser descrito por acreditar-se que as EOA espontâneas podem influenciar nas respostas das EOAT na orelha direita, uma vez que apesar de ser geralmente bilaterais, ao se apresentar unilateralmente, são mais frequentes na orelha direita como pode ser observado em outras pesquisas $(12,13)$.

Vale ressaltar que a média geral das amplitudes da orelha direita $(10,22 \mathrm{~dB})$ e esquerda $(9,48 \mathrm{~dB})$ não apresentou diferença estatisticamente significativa podendo também ser observado em vários estudos $(5,7,11,14,15)$.

Ao compararmos as respostas das EOAT na primeira e na segunda testagem verificou-se prevalência de maiores amplitudes de respostas das EOAT antes da jornada de trabalho, sendo que na orelha direita, $97,5 \%$ apresentaram melhores respostas na primeira testagem, e na orelha esquerda foram $88,6 \%$. Desse modo a amplitude geral frequentemente foi melhor na primeira testagem e pior na segunda testagem após a exposição ao ruído, como tam-
Tabela 3. Variaçãoem média da amplitudegeral das EOATem $\mathrm{dB}$ nas orelhas direita e esquerda.

\begin{tabular}{lcc}
\hline EOAT & $\mathrm{OD}(\mathrm{dB})$ & $\mathrm{OE}(\mathrm{dB})$ \\
\hline I $^{\text {a}}$ testagem & 10,22 & 9,48 \\
$2^{\text {a }}$ testagem & 9,80 & 8,79 \\
Variação & 0,42 & 0,69 \\
\hline
\end{tabular}

bém pode ser observado na literatura pesquisada $(5,6,7$, $8,9,10,11)$

$\mathrm{Na}$ análise das EOAT observou-se que $40 \%$ apresentaram ausência bilateral e $60 \%$ unilateral. Em contrapartida FioRINI verificou ausência predominantemente bilateral em 48,7\% dos casos e apenas 20\% de ausência unilateral (11).

Dos que apresentaram ausência de resposta em pelo menos uma orelha, nove (18,36\%) falharam na orelha direita e cinco $(10,2 \%)$ na orelha esquerda após a exposição ao ruído. Fiorini também referiu maior prevalência de ausência de EOAT para orelha direita em trabalhadores expostos ao ruído (11), em contrapartida, Souza encontrou maior ausência nas EOAT para orelha esquerda (6).

No que diz respeito à reprodutibilidade coclear foi analisada a variação da média da amplitude geral entre a primeira e a segunda testagem, sendo $0,42 \mathrm{~dB}$ para orelha direita e 0,69 dB para orelha esquerda. Tal fato também pode ser observado em um estudo no qual os pesquisadores encontraram variações das amplitudes de resposta geral com valores menores que $1 \mathrm{~dB}$ (7). Entretanto a literatura evidenciou que houve redução significativa no nível das EOAT após exposição ao ruído, sendo os valores maiores que $1 \mathrm{~dB}$ (8). Outro estudo ainda relatou valores acima de $1,7 \mathrm{~dB}(5)$.

De todos os trabalhadores que apresentaram ausência de resposta em pelo menos uma orelha, nenhum deles mencionou dificuldade em escutar. Esse dado sugere que mesmo após a exposição ao ruído, os trabalhadores não apresentaram sintomas auditivos perceptíveis. Na literatura pesquisada, foi observado que a ausência de resposta das EOAT sugere lesão inicial das células ciliadas externas, sendo assim o sintoma muitas vezes é imperceptível, porém já evidencia alteração da membrana basilar e esta pode ser identificada precocemente por meio das EOAT (16).

Os 10 trabalhadores que apresentaram ausência de resposta em pelo menos uma orelha possuíam idade média de 35,3 anos e tempo de exposição ao ruído de 7,8 anos. Entretanto, não foi encontrada diferença estatisticamente significante entre tempo de exposição, idade, exposição a produto químico e ausência de emissões. 
Dos 49 trabalhadores pesquisados, apenas cinco $(10,2 \%)$ relataram presença de zumbido, sendo que não houve diferença estatisticamente significativa entre presença de zumbido e ausência de emissões. Em contrapartida, na literatura pesquisada, há uma alta prevalência de zumbido na população exposta a ruído ocupacional $(17,18)$.

\section{CONCLUSÃO}

Quanto à análise comparativa da amplitude geral das EOAT antes a após a atividade laborativa observou-se:

- Maior variação da amplitude na orelha esquerda $(0,69$ dB) em relação à orelha direita $(0,42 \mathrm{~dB})$;

- Verificaram-se melhores respostas antes da atividade ocupacional: $97,5 \%$ na orelha direita e $88,6 \%$ na orelha esquerda.

- Tanto na primeira como na segunda testagem a orelha direita apresentou maior amplitude máxima em relação à orelha esquerda.

Quanto à análise das respostas obtidas na segunda testagem das EOAT:

- 20,4\% dos trabalhadores apresentaram EOAT ausentes em pelo menos uma das orelhas. Destes, 100\% usou proteção auricular em empresas anteriores e utilizam atualmente, $50 \%$ trabalha com produtos químicos, apenas $10 \%$ pratica atividade extra-ocupacional com elevado nível de pressão sonora e nenhum deles informou dificuldade em escutar.

Diante dos achados obtidos observou-se que o exame de EOAT de fato é um instrumento que possibilita o monitoramento de variações no funcionamento das CCE, mesmo em indivíduos com limiares auditivos dentro do padrão de normalidade. Entretanto a análise dos resultados evidenciou que não houve dados estatisticamente significantes no que se refere aos critérios adotados: idade, tempo de exposição, exposição a produtos químicos e atividade extra- ocupacional com elevado nível de pressão sonora.

\section{REFERÊNCIAS BIBLIOGRAFICAS}

1. Brasil. Ministério da Saúde, Secretaria de atenção à saúde, Departamento de ações programáticas estratégicas. Protocolo de complexidade diferenciada: Perda auditiva induzida por ruído. $1^{\underline{a}}$ ed. Brasília: Editora do ministério da Saúde, 2006. 40p. Disponível em 19 de Out. 2010.

2. Seligman J. Sinais e sintomas na PAIR. In NudelmanN AA, Costa EA, Seligman J, Ibañez RN. PAIR- Perda Auditiva Induzida pelo Ruído. Vol.I, Porto Alegre: Bagaggem Comunicação, 1997. p. 143-151.
3. Sousa LCA, Piza MRT, Alavarenga KF, Coser PL. Emissões Otoacústicas. Eletrofisiologia da Audição e Emissões Otoacústicas: Princípios e Aplicações Clínicas. São Paulo: Tecmedd; 2008, p. 109-30.

4. Lewis DR. Emissões Otoacústicas: Aplicações clínicas. In Ferreira R.P., Befi-lopes DM, Limongi SCO. Tratado de fonoaudiologia. São Paulo: Roca; 2004. p. 617-629.

5. Barros SMS, Frota S, Atherino CCT, Osterne F. A eficiência das emissões otoacústicas transientes e audiometria tonal na detecção de mudanças temporárias nos limiares auditivos após exposição a níveis elevados de pressão sonora. Rev Bras Otorrinolaringol. 2007, 73(5):592-8.

6. Souza DV. Estudo comporativo das Emissões Otoacústicas em militares expostos e não expostos ao ruído. 2009. 170 f. Dissertação de Mestrado (Mestrado em fonoaudiologia) - Universidade Veiga de Almeida, Rio de Janeiro, 2009.

7. Negrão MA, Soares E. Variações nas amplitudes de respostas das emissões otoacústicas evocadas e suscetibilidade à perda auditiva induzida por ruído - PAIR. Rev Cefac. 2004, 6(4):414-22.

8. Sliwinska-kowalska M, KotyloP, Hendler B. Comparandose as alterações transitórias, em Emissões Otoacústicas e audiometria tonal liminar, após a exposição ao ruído industrial curto. Vol 1, Ed.2. Lodz, Polônia: Noise \& Health, p. 50-57, 1999.

9. Marques FP, Costa EA. Exposição ao ruído ocupacional: alterações no exame de emissões otoacústicas. Rev Bras Otorrinolaringol. 2006, 72(3):362-6.

10. Mor R, Frasson MA. Emissões otoacústicas e sistema olivococlear medial: pacientes com zumbido sem perda auditiva. Rev Pró-fono. Barueri. 2005, 17(3).

11. Fiorini AC, Fischer FM. Expostos e não expostos a Ruído Ocupacional: Estudo dos Hábitos sonoros, Entalhe Audiométrico e Teste de Emissões Otoacusticas Evocadas por estimulo transiente. Rev. Distúrbios da Comunicação. São Paulo, vol. 16, n. 3, p. 371-383, Dez 2004.

12. Azevedo MF. Emissões Otoacústicas. In: Figueiredo MS. Conhecimentos essenciais para conhecer bem emissões e bera. São José dos Campos: Pulso; p. 35-75. 2003.

13. Nodarse EM. Empleo de las emisiones otoacusticas para el pesquisaje del deficit auditivo. Rev Habanera Cienc Méd. 2006, 5(1).

14. Konopka W, Pawlaczyk-Luszczynska M, SliwinskaKowalska M, Grzanka A, Zalewski P. Effects of impulse noise 
on transiently evoked otoacousticemission in soldiers. Int J Audiol. 2005, 44(1):3-7.

15. Olszewski J, MiBoDski J, Olszewski S, Majak J. et al. Hearing threshold shift measured by otoacoustic emissions alter shooting noise exposure in soldiers using hearing protectors. Otolaryngology -head and Neck Surgery. 2007, 136(1):78-81.

16. Jerger S, Jerger J. Alterações Auditivas: Um Manual para Avaliação Clínica. Rio de Janeiro, Livraria Atheneu Editora, 1989.
17. Dias A, Cordeiro R. Interação entre grau de perda auditiva e o incômodo com zumbidos em trabalhadores com história de exposição ao ruído. Rev Bras de Otorrinolaringol. 2008, 74(6):876-8.

18. Possani LNA. Estudo da Prevalência e das características do zumbido em trabalhadores expostos a o ruído. 2006. 109 f. Dissertação (Mestrado em Epidemiologia)- Universidade Federal do Rio Grande do Sul- UFRGS, Porto Alegre.

Anexo.

\section{Termo de consentimento livre e esclarecido}

O Sr(a). está sendo convidado (a) a participar da pesquisa que se intitula "Emissões Otoacústicas como Instrumento de Vigilância Epidemiológica na Saúde do Trabalbador"

O objetivo deste estudo é analisar alterações cocleares através das otoemissões acústicas evocadas transientes em indivíduos expostos ao risco combinado: ruído e produtos químicos.

Caso aceite participar como sujeito desta pesquisa, o (a) Sr. (a) irá se submeter a responder uma anamnese e realizar o exame de otoemissões acústicas transientes.

Não existem benefícios médicos diretos para o sujeito deste estudo. Entretanto os resultados deste estudo podem ajudar os pesquisadores a verificar se o ruído causa mínimas alterações na cóclea (orelha interna) da população exposta ao ruído ocupacional. Não existem riscos médicos ou desconfortos associados com este projeto.

O participante desta pesquisa não passará por nenhum procedimento invasivo. O exame de otoemissões acústicas trata-se de um exame indolor, rápido (aproximadamente cinco minutos para realização). O exame não trás nenhum tipo de trauma ou lesão. Será inserida uma sonda em cada ouvido separadamente, em seguida serão emitidos sons (pequenos apitos). Uma das recomendações é que o participante da pesquisa permaneça quieto durante a testagem.

Por ser um exame objetivo, o participante não precisa responder a testagem, sendo que o resultado é fornecido pelo equipamento. O exame será realizado em dois momentos, antes da atividade laborativa, com repouso acústico de no mínimo 14 horas e após a jornada de trabalho. Após a primeira testagem o participante da pesquisa seguirá normalmente para sua função de trabalho diário sem nenhum tipo de restrição. Será solicitado o retorno no final da atividade laborativa para realização novamente do exame (EOA). Em seguida será liberado e fornecido o resultado dos exames realizados.

Fica claro que sua participação é voluntária, não sendo obrigado a responder a anamnese ou fazer o exame, mesmo que já tenha assinado o consentimento de participação. Se desejar, poderá retirar seu consentimento a qualquer momento e isto não trará nenhum prejuízo ao seu atendimento.
Os pesquisadores não pagarão nenhum valor em dinheiro ou qualquer outro bem pela sua participação, assim como o Sr. (a) não terá nenhum custo adicional. Os seus dados serão mantidos em sigilo. Serão analisados em conjunto com os de outros pacientes e não serão divulgados dados de nenhum paciente isoladamente. O (a) Sr. (a) poderá esclarecer suas dúvidas durante toda a pesquisa com as pesquisadoras, da Universidade Federal de Sergipe Luana Araujo dos Santos (88011391) e Any Caroline Aragão, ou com a Professora responsável Ms. Priscila Oliveira (9131.2569). As pesquisadoras comprometem-se a utilizar os dados coletados somente para os fins que se destinam, ou seja, pesquisa.

Acredito ter sido suficientemente informado (a) a respeito das informações que li ou que foram lidas por mim, descrevendo a pesquisa intitulada "Emissões Otoacústicas como Instrumento de Vigilância Epidemiológica na Saúde do Trabalhador"

Eu discuti com as fonoaudiólogas Luana Araujo dos Santos e Any Caroline Aragão sobre a minha decisão em participar do estudo. Ficaram claros para mim quais são os propósitos do estudo, os procedimentos a serem realizados, seus desconfortos e riscos, as garantias de confidencialidade e de esclarecimentos permanentes. Ficou claro também que minha participação é isenta de despesas. Concordo voluntariamente em participar deste estudo e poderei retirar o meu consentimento a qualquer momento, antes ou durante o mesmo, sem penalidades ou prejuízo, ou perda de qualquer benefício que eu possa ter adquirido, ou no meu atendimento neste serviço.

Nome do paciente

Assinatura do paciente Data

Assinatura da testemunha Data

Declaro que obtive de forma apropriada e voluntária o Consentimento Livre e Esclarecido deste paciente para a participação neste estudo.

Assinatura do responsável pelo estudo Data 